\title{
Carrier Rate of Citrullinemia, Type I in Individual Ethnic Groups Using an Expanded Carrier Test
}

\author{
Jonah Bardos ${ }^{1 *}$, Catherine Terhaar ${ }^{2}$, Natalia Echeverri ${ }^{1}$ and Ryan E Longman ${ }^{1}$ \\ ${ }^{1}$ University of Miami, USA \\ ${ }^{2}$ Progenity, Ann Arbor, USA
}

*Corresponding author: Jonah Bardos, Department of OBGYN, University of Miami, Miller School of Medicine, USA.

Received Date: March 13, 2019

Published Date: March 18, 2019

\begin{abstract}
Purpose: To identify population-specific carrier frequencies for Citrullinemia Type 1. Better knowledge of population specific carrier frequencies improves carrier testing and genetic counseling; however, prior reports have not captured data in specific ethnic groups. Identifying a fetus at risk for CTLN1 could allow prenatal planning by arranging for appropriate post-partum care to mitigate or prevent long lasting neurological damage with prompt use of metabolic therapy.
\end{abstract}

Methods: Retrospective review of 11,132 individuals who underwent expanded carrier testing utilizing a genotyping panel. The CTLN1 variants included were the common $1168 \mathrm{G}>\mathrm{A}$ variant, and the rarer $421-2 \mathrm{~A}>\mathrm{G} / 910 \mathrm{C}>\mathrm{T}$ variant. Adjusting for the genotyping panel's detection rate we calculated the carrier frequency for specific ethnic groups.

Results: Pan-ethnic carrier frequency of 1:383 $(10 * 2.9 / 11,132)$, corresponding to a population prevalence of $1: 575,000$. Adjusted carrier rates are: 1/124 in the Ashkenazi Jewish group; 1/392 in the Hispanic group;1/422 in the Caucasian/White group.

Conclusion: As expanded carrier testing utilization increases, it is important to continue to reassess carrier and disease frequencies of rare conditions with large ethnically diverse cohorts. This is the first report, to our knowledge, to document CTLN1 carrier rate in individual ethnic groups. This knowledge can guide preconception and prenatal counseling enabling patients and providers to identify resources and neonatal management options.

Precis: CTLN1 carrier rates vary amongst different ethnic groups

Keywords: Citrullinemia type 1; Carrier rate; Ethnic groups

\section{Introduction}

Citrullinemia type I (CTLN1) is an autosomal recessive urea cycle disorder characterized by an arginosuccinate synthetase (ASS1 EC 6.3.4.5) enzyme deficiency. ASS1 is a urea cycle enzyme predominantly expressed in periportal hepatocytes which catalyzes the formation of arginosuccinate from citrulline and aspartate [1-3]. Type 1, or classical citrullinemia (CTLN1: OMIM\#215700) is caused by pathogenic variants in the ASS gene, most commonly a missense in exon 15 of $1168 \mathrm{G}>\mathrm{A} .2$ The presence of this variant decreases ASS1 enzyme function, leading to the accumulation of citrulline and hyperammonemia [4,5]. Effects of this condition can be devastating, with long-term neurological deficits including spasticity, seizures, loss of consciousness, and death. 6 Identifying a fetus at risk for CTLN1 could allow prenatal planning by arranging for appropriate post-partum care to mitigate or prevent long lasting neurological damage with prompt use of metabolic therapy [7].

The prevalence of CTLN1 is estimated at 1 in 57,000 live births.7 Previously published papers out of the United States reported a wide-ranging prevalence between $1: 36,000$ to $1: 250,000$ or a carrier frequency ranging from 1:95 to 1:250 [8-10]. Knowledge of population-specific carrier frequencies improves carrier testing and genetic counseling; however, prior reports have not captured data in specific ethnic groups.

\section{Materials and Methods}

We performed a retrospective review of 11,132 individuals who underwent expanded carrier testing utilizing a genotyping 
panel. The CTLN1 variants included were the common 1168G>A variant, and the rarer $421-2 \mathrm{~A}>\mathrm{G} / 910 \mathrm{C}>\mathrm{T}$ variant [2]. To adjust for the genotyping panel's detection rate of $35 \%$ for CTLN1, the number of carriers for each group was multiplied by 2.9 (100/35). We calculated the overall carrier rate of CTLN1 and for individual ethnic groups.

\section{Results}

Our data demonstrate a pan-ethnic carrier frequency of 1:383 $(10 * 2.9 / 11,132)$, corresponding to a population prevalence of 1 : 575,000 (Table 1). The positivity rates for individual ethnic groups were Ashkenazi Jewish (1/361), Caucasian/White (4/4,899), Hispanic $(1 / 1,137)$, Other or Mixed $(4 / 2,784)$. When positivity rates are adjusted for the $35 \%$ detection rate, the carrier rates are: 1/124 in the Ashkenazi Jewish group; 1/392 in the Hispanic group;1/422 in the Caucasian/White group; and 1/240 in the Other or Mixed group. No carriers were identified in the African American/Black (785 tests), Asian (1,091 tests), Native American (16 tests), or Sephardic Jewish ethnic groups (58 tests). As expected, 9 patients were positive for the 1168G $>$ A variant and one patient who identified as Other or Mixed was positive for the 421$2 \mathrm{~A}>\mathrm{G}$ variant.

Table 1: Citrullinemia Type I carrier rates.

\begin{tabular}{|c|c|c|}
\hline Ethnicity & Positive Tests & Adjusted Carrier Rates \\
\hline Ashkenazi Jewish & $1 / 361$ & $1 / 124$ \\
\hline Caucasian/White & $4 / 4,899$ & $1 / 422$ \\
\hline Hispanic & $1 / 1,137$ & $1 / 392$ \\
\hline Other or Mixed & $4 / 2,784$ & $1 / 240$ \\
\hline African American/Black & $0 / 785$ & - \\
\hline Asian & $0 / 1,091$ & - \\
\hline Native American & $0 / 16$ & - \\
\hline Sephardic Jewish & $0 / 58$ & - \\
\hline Overall & $10 / 11,132$ & $1 / 383$ \\
\hline
\end{tabular}

\section{Discussion}

Prior studies have reported varying incidence of citrullinemia in specific populations; however, this is the first report, to our knowledge, to document CTLN1 carrier rate in individual ethnic groups. We report a general population carrier rate of $1 / 383$, which is slightly lower than expected based on reported disease frequencies. Possible explanations for this difference include an overestimate of disease prevalence reported in the literature (which may explain the wide-ranging prevalence that is reported) or an overestimate of the frequency of variants included in this assay.

\section{Conclusion}

As expanded carrier testing utilization increases, it is important to continue to reassess carrier and disease frequencies of rare conditions with large ethnically diverse cohorts. This will provide better understanding of general population risk and more accurate reporting of residual risk given a negative result. The knowledge of carrier status can guide preconception and prenatal counseling by identifying individuals at risk to have offspring with genetic disease and enables patients and providers to identify resources and understand neonatal management options.

\section{Acknowledgment}

Data for this was provided by Progenity, Inc., in collaboration with the University of Miami.

\section{Conflict of Interest}

No conflict of interest.

\section{References}

1. Laróvere LE, Angaroni CJ, Antonozzi SL, Bezard MB, Shimohama M, et al. (2009) Citrullinemia Type I, Classical Variant. Identification of ASS p G390R (c.1168G>A) mutation in families of a limited geographic area of Argentina: A possible population cluster. Clin Biochem 42(10-11): 11661168.

2. Engel K, Höhne W, Häberle J (2008) Mutations and polymorphisms in the human argininosuccinate synthetase (ASS1) gene. Hum Mutat 30(3): 300-307.

3. Woo HI, Park HD, Lee YW (2014) Molecular genetics of citrullinemia types I and II. Clin Chim Acta 431: 1-8.

4. Saheki T, Nakano K, Kobayashi K, Imamura Y, Itakura Y, et al. (1985) Analysis of the enzyme abnormality in eight cases of neonatal and infantile citrullinaemia in Japan. J Inherit Metab Dis 8(3): 155-156.

5. Kobayashi K, Kakinoki H, Fukushige T, Shaheen N, Terazono H, et al. (1995) Nature and frequency of mutations in the argininosuccinate synthetase gene that cause classical citrullinemia. Hum Genet 96(4): 454-463.

6. Quinonez SC, Thoene JG (2004) Citrullinemia Type I. Gene Reviews ${ }^{\circledR}$, University of Washington, Seattle, USA.

7. Summar M (2001) Current strategies for the management of neonatal urea cycle disorders. J Pediatr 138(1 Suppl): S30-S9.

8. Brusilow SW, Horwich AL (2001) Urea cycle enzymes. Scriver CR, Beaudet AL, Sly WS, Valle D, Childs B, et al. (edts), The Metabolic \& Molecular Bases of Inherited Diseases ( $8^{\text {th }}$ edn) New-York, McGraw-Hill, USA.

9. Summar ML, Endo F, Kölker S (2014) On the Creation, Utility and Sustaining of Rare Diseases Research Networks: Lessons learned from the Urea Cycle Disorders Consortium, the Japanese Urea Cycle Disorders Consortium and the European Registry and Network for Intoxication Type Metabolic Diseases. Molecular Genetics and Metabolism 113(1-2): 105-108.

10. Marsden D (2003) Expanded newborn screening by tandem mass spectrometry: the Massachusetts and New England experience. Southeast Asian J Trop Med Public Health 34 Suppl 3: 111-114 